\title{
Stochastic 3D modeling of complex three-phase microstructures in SOFC-electrodes with completely connected phases
}

\author{
Matthias Neumann ${ }^{\mathrm{a}, *}$, Jakub Staněk ${ }^{\mathrm{b}}$, Omar M. Pecho ${ }^{\mathrm{c}, \mathrm{d}}$, Lorenz Holzer ${ }^{\mathrm{d}}$, \\ Viktor Beneše ${ }^{\mathrm{e}}$, Volker Schmidt ${ }^{\mathrm{a}}$ \\ ${ }^{a}$ Institute of Stochastics, Ulm University, D-89069 Ulm, Germany \\ ${ }^{b}$ Department of Mathematics Education, Faculty of Mathematics and Physics, Charles University \\ Prague, CZ-18675 Prague, Czech Republic \\ ${ }^{c}$ Institute for Building Materials, ETH Zurich, CH-8093 Zurich, Switzerland \\ ${ }^{d}$ Institute of Computational Physics, ZHAW Winterthur, CH-8400 Winterthur, Switzerland \\ ${ }^{e}$ Department of Probability and Mathematical Statistics, Faculty of Mathematics and Physics, Charles \\ University Prague, CZ-18675 Prague, Czech Republic
}

\begin{abstract}
A parametric stochastic 3D model for the description of complex three-phase microstructures is developed. Such materials occur for example in anodes of solid oxide fuel cells (SOFC) which consist of pores, nickel (Ni) and yttriastabilized zirconia (YSZ). The model is constructed using tools from stochastic geometry. More precisely, we model the backbones of the three phases by a certain class of random geometric graphs called beta-skeletons. This allows us to reproduce complete connectivity of all three phases as observed in experimental image data of a pristine Ni-YSZ anode as well as the prediction of volume fractions by model parameters. Finally a slightly generalized version of this model enables a good fit to experimental image data with respect to transport relevant microstructure characteristics and the length of triple phase boundary. Model validation is performed by comparing effective transport properties from finite element (FE) simulations based on 3D-data from the stochastic model and from tomography of real Ni-YSZ anodes. Moreover, the virtual, but realistic Ni-YSZ microstructures can be used for investigating the quantitative influence of microstructure characteristics on various physical properties and consequently on the performance of the
\end{abstract}

\footnotetext{
*Corresponding author. Phone: +49 73150 23617. Fax: +49 73150 23649. Email: matthias.neumann@uni-ulm.de.
}

Preprint submitted to Computational Material Science

February 29, 2016

(C) 2016. This manuscript version is made available under the Elsevier user license http://www.elsevier.com/open-access/userlicense/1.0/ 
anode material.

Keywords: 3D microstructure model, Beta-skeleton, Effective transport properties, Nickel-YSZ electrode, Solid oxide fuel cell, Stochastic geometry.

\section{Introduction}

Compared to conventional electricity generation the use of solid oxide fuel cells (SOFC) leads to an improvement with respect to efficiency, reliability and environmental impact. The electrodes of SOFC consist of materials 5 which allow electric, ionic, and gas transport. For this purpose porous composites of nickel (Ni) and yttria-stabilized zirconia (YSZ) are widely used. In Ni-YSZ anodes oxygen ions and hydrogen are transported through the YSZ phase and the pores, respectively, to the triple phase boundary (TPB), where the chemical reaction resulting in free electrons takes place. The free electrons are then transported through the Ni phase to the metallic interconnector. It has been shown that properties of mass transport and charge transfer in the anode and thus the performance of the SOFC are significantly influenced by the Ni-YSZ microstructure, see e.g. [27]. Since the Ni-YSZ microstructure is rather complex one is interested in relating its influence on 15 transport processes to a few microstructure characteristics. A possible approach for investigating the relationship between microstructure characteristics and properties of mass transport as well as charge transfer is based on 3D imaging of Ni-YSZ microstructures because 3D imaging allows the computation of well-defined microstructure characteristics, e.g. volume fractions, geometric tortuosities, length of TPB. The corresponding transport properties like effective conductivity in $\mathrm{Ni}$ and YSZ can be obtained by numerical simulations, e.g. by the finite element (FE) or the lattice Boltzmann method. This approach enables a direct investigation of microstructure characteristics and effective transport properties [14, 28, 32]. However, it has the disadvantage that due to the high costs of 3D imaging the amount of experimental image data is limited, which is a barrier for a systematical investigation of quantitative relationships between microstructure characteristics and the corresponding effective materials properties.

An alternative approach is based on stochastic 3D modeling for the generation of virtual microstructures. These virtual microstructures are used as an input for numerical simulations and finally the microstructure characteristics of the virtual structures can be related to the corresponding transport prop- 
erties. In case that the stochastic model generates virtual microstructures in short time and the microstructure characteristics can be systematically varied, the combination of stochastic microstructure simulations and numerical transport simulations provides a large database for an investigation of the 5 microstructure influence on physical processes.

During the last years, various models for the generation of virtual Ni-YSZ anodes have been developed, where both the Ni- and YSZ phases are represented as a union of monodispersed [3, 12] or polydispersed balls $[5,16,20]$. In these models the microstructures are iteratively generated by the aid of different sphere-packing algorithms. A generalization to ellipsoidal and cylindrical particles was recently done in [2] and [26]. However, the existing microstructure models are not able to reproduce more complex shapes [27], and the connectivity properties observed in experimental image data [15]. While these approaches allowed qualitative conclusions about the relationships between 15 microstructure characteristics and physical properties, the quantification of these relationships and their applicability to real materials are still unclear.

Thus, in the present paper, we develop a parametric stochastic 3D microstructure model for generating three-phase microstructures with completely connected phases, which can be applied e.g. to simulate the 3D microstructure of certain pristine porous Ni-YSZ anodes in short time. This model is different from the existing ones because the microstructure is directly constructed without any iterative procedure. The presented model is based on methods from stochastic geometry, which have been successfully applied for various kinds of microstructure modeling, see [4] and the references therein. Note that the model is not particle-based and it is possible to generate a wide spectrum of virtual microstructures, where all three phases are completely connected with probability 1 . In particular, the model parameters are fitted to experimental image data in which the three phases are completely connected. The fit is done with respect to volume fraction, geodesic tortuosity, constrictivity measuring bottleneck effects within the phases, and the length of TPB. Furthermore, the model is validated by comparing effective conductivities of experimental image data with those of the fitted virtual microstructures for $\mathrm{Ni}$ - and YSZ phases, where effective conductivities are simulated using the FE-method. In a forthcoming paper the parameters of the stochastic microstructure model will be systematically varied in order to obtain a large database for investigating the quantitative relationships between microstructure characteristics and effective physical properties of Ni-YSZ anodes, as it was done for two-phase microstructures in [10] and 
[29]. With the effective transport properties and the length of TPB it is possible to simulate the area specific resistance (ASR) of the anode by the model proposed in [8], which allows to study the microstructure influence on the ASR. Using this approach we intend to utilize virtual materials design in

5 finding an optimal constellation of parameters of the stochastic microstructure model with respect to performance and lifetime of the electrode in order to better understand the geometry of such an optimal structure.

The paper is organized as follows. In Section 2 the microstructure characteristics considered in the present paper are described. The stochastic microstructure model is introduced in Section 3, where some general properties of the beta-skeleton are also discussed. After fitting the parameters of the stochastic model to experimental image data and a validation of the model in Section 4, Section 5 concludes the paper.

\section{Microstructure characteristics}

15

The 3D microstructure of porous Ni-YSZ anodes is considered in order to better understand the influence of the microstructure on the overall performance of the cell. The challenge of parametric modeling of these three-phase microstructures is to achieve a good fit with respect to the properties of real materials (having the same phase composition). The properties that have to be fitted are the transport relevant microstructure characteristics and the specific length of TPB. As the most relevant transport characteristics, following the argumentation in [10] and [14], we consider the volume fractions of the three phases, the lengths of transport paths through the material and the constrictivities, which measure the width of bottlenecks in a microstructure. Additionally we consider two further microstructure characteristics: The distribution of so-called chordlengths for measuring anisotropy effects in Section 4.1, and the specific surface area between two of the three phases for discussing limitations of the model in Section 4.4.

For each parameter constellation of the stochastic microstructure model the model-based characteristics are expected values, i.e., microstructure characteristics of an observed sample are considered as random variables and they are estimators for the model-based microstructure characteristics as shown in Figure 1. It is reasonable to consider microstructure characteristics as random variables since experimental image data represents only a cut-out of the complete anode microstructure. Thus microstructure characteristics are random in the sense that they depend on the position at which the im- 


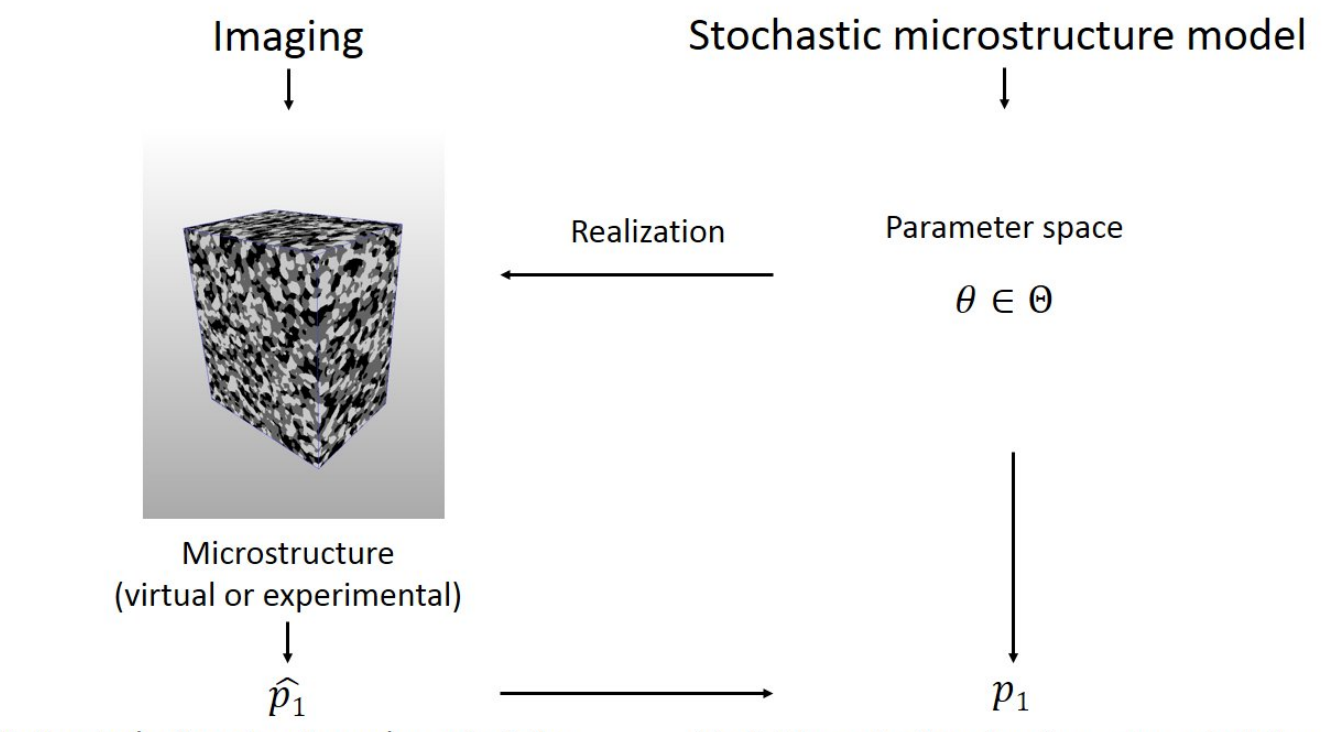

Estimated microstructure characteristics Model-based microstructure characteristics

Figure 1: Interplay between model-based and estimated microstructure characteristics.

age is taken. For the same reason we consider the three phases (pores, YSZ and $\mathrm{Ni}$ ) as stationary random sets denoted by $\Xi_{1}, \Xi_{2}, \Xi_{3}$, see [4]. Note that the boundary between two phases is considered to belong to both neighboring phases. The cutouts of the random sets $\Xi_{1}, \Xi_{2}, \Xi_{3}$ within a rectangular 5 cuboid $W=\left[0, w_{1}\right] \times\left[0, w_{2}\right] \times\left[0, w_{3}\right]$, where $w_{1}, w_{2}, w_{3} \in \mathbb{N}$ are some natural numbers, form the Ni-YSZ microstructure in $W$. In this section we describe the microstructure characteristics of a stationary random set $\Xi$.

When we fit the stochastic microstructure model to experimental image data in Section 4 we intend to minimize the difference between microstructure characteristics estimated from image data and the ones estimated on the basis of realizations of the model.

\subsection{Volume fraction}

The volume fraction of a stationary random set $\Xi$ is defined by

$$
p=\mathbb{E} \nu_{3}(\Xi \cap W) / \nu_{3}(W),
$$

where $\nu_{3}$ denotes the three-dimensional Lebesgue-measure, i.e. the volume 15 in $3 \mathrm{D}$. Note that $p$ does not depend on the specific choice of the observation window $W$. The experimental 3D image data provides information on a 3D 
grid. Thus we use the point count-method, see [4], for the estimation of $p$, i.e., we count the number of all grid points belonging to $\Xi$ and divide it by the number of all grid points. This leads to an unbiased estimator of $p$.

\subsection{Mean geodesic tortuosity}

${ }_{5} \quad$ Mean geodesic tortuosity $\tau_{W}$ in the observation window $W$ is defined as the expected length of a path going through $\Xi \cap W$ in transport direction from one side of the anode to the other side divided by the material thickness. Note that in analyzing microstructures there exist many different concepts of tortuosity. For an overview we refer to [6]. The concept of geodesic tortuosity, which we use here, is also used in [29] for the prediction of effective transport properties by microstructure characteristics. To estimate $\tau_{W}$ from image data, we approximate the path lengths in transport direction on the voxel grid, see Figure 2. For this purpose we use Dijkstra's algorithm, see e.g. [30].

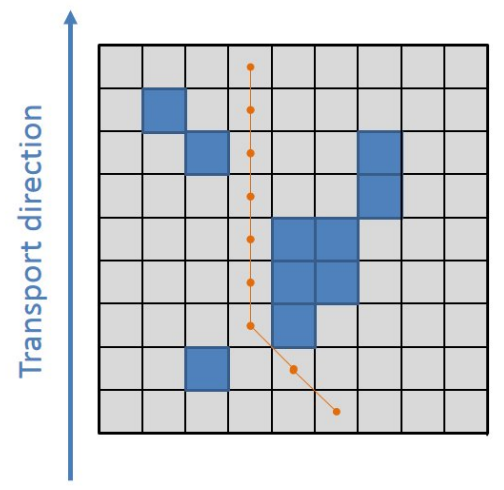

Figure 2: Approximation of a shortest path on a grid. The transport path is represented in orange. Note that $2 \mathrm{D}$ structures are shown for a better visualization, while all computations are done in $3 \mathrm{D}$.

\subsection{Constrictivity}

As it was already mentioned in Section 1, constrictivity in the observation window $W$ denoted by $\beta_{W}$ is a measure for bottleneck effects in a microstructure, which is based on the concept of the so-called continuous phase size distribution and the geometrical simulation of mercury intrusion porosimetry [22]. The strong influence of this microstructure characteristic on effective transport properties in porous microstructures was recently shown in [10] and [29]. 


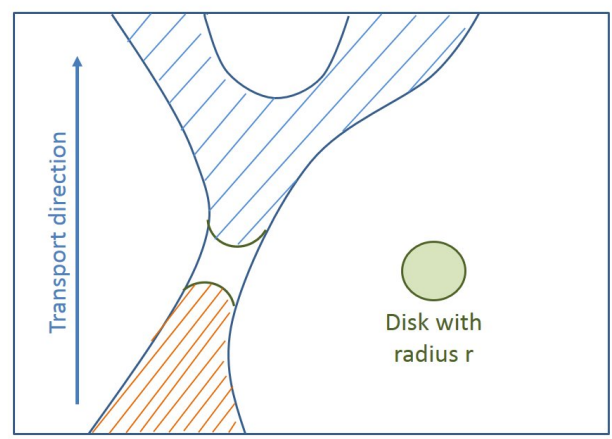

Figure 3: The union of the blue and orange subsets is the part of the considered phase that can be covered by disks of radius $r$ such that these disks are completely contained in the set. The orange subset is the part that can be filled by disks with radius $r$ in transport direction.

Constrictivity $\beta_{W}$ of a stationary random set $\Xi$ in $W$ is formally defined as the squared ratio $\beta_{W}=\left(r_{\min , W} / r_{\max , W}\right)^{2}$. Here $r_{\max , W}$ is the maximum radius $r$ such that in expectation at least $50 \%$ of $\Xi \cap W$ can be covered by balls of radius $r$, where these balls are completely contained in $\Xi \cap W$.

${ }_{5}$ Furthermore, $r_{\min , W}$ is the maximum radius $r$ such that in expectation at least $50 \%$ of $\Xi \cap W$ can be filled by an intrusion of balls with radius $r$ in transport direction, see Figure 3. Since the intrusion in transport direction determining $r_{\min , W}$ is strongly influenced by bottlenecks in $\Xi \cap W$ while $r_{\max }$ is not, it holds that:

(a) $r_{\min , W} \leq r_{\max , W}$ and thus $0 \leq \beta_{W} \leq 1$,

(b) if $\beta_{W}$ is close to 0 , then there are many narrow constrictions in $\Xi \cap W$ and

(c) if $\beta_{W}=1$, then there are no constrictions at all.

To estimate $\beta_{W}$ from image data we use the algorithm described in [22].

\subsection{Specific length of triple phase boundary}

The specific length $\delta$ of TPB is the length of TPB per unit volume, mathematically defined as

$$
\delta=\frac{1}{\nu_{3}(W)} \mathbb{E} \mathcal{H}_{1}\left(\Xi_{1} \cap \Xi_{2} \cap \Xi_{3} \cap W\right),
$$


where $\mathcal{H}_{1}$ denotes the one-dimensional Hausdorff-measure in $\mathbb{R}^{3}$, i.e. the length of a one-dimensional (rectifiable) object in 3D. Note that $\delta$ does not depend on the choice of the specific observation window $W$. The specific length $\delta$ of TPB is an important characteristic for porous Ni-YSZ anodes 5 since the chemical reaction resulting in free electrons takes place at the TPB. Thus an increase of the length of TPB in the anode leads to a higher performance of the cell. For more information about the role of TPB in Ni-YSZ microstructures the reader is referred to [27].

For estimating $\delta$ from image data we use two different methods. The first estimator denoted by $\widehat{\delta}_{1}$ just counts configurations of 8 neighboring voxels in the image which contain voxels from each of the three phases. The estimator $\widehat{\delta_{1}}$ can be easily computed, but it is biased since we do not take into account the spatial arrangement of TPB voxels. As a second estimation of $\delta$ we use a more elaborate algorithm. The corresponding estimator is denoted by $\widehat{\delta_{2}}$. 15 For computing $\widehat{\delta_{2}}$ we skeletonize the TPB. Then $\widehat{\delta_{2}}$ is defined as the specific length of this skeleton.

In Section 4.2 we fit the parameters of our stochastic microstructure model to experimental image data by an iterative optimization method, where the cost function depends on relative deviations between microstructure characteristics of experimental and simulated data. Here $\delta$ has to be estimated in each iteration step. Thus we compute the cost function with respect to the estimator $\widehat{\delta_{1}}$. In Section 4.3 we validate the model by comparing the estimators $\widehat{\delta}_{2}$ computed on the basis of experimental image data and simulated data with fitted parameters. It should be noticed that a connected compoall three phases of the considered TPB component are connected with their 'base'. For example, the Ni phase at the TPB must be connected with the current collector in order to enable harvesting of electrons produced by the TPB-reaction. Similarly the YSZ phase must be connected with the electrolyte to provide oxygen ions for the TPB-reaction. In real microstructures, degradation often leads to loss of connectivity and hence active and inactive TPB have to be distinguished. In the present work, however, we intend to simulate microstructures of pristine anodes, where all three phases are completely percolating. Therfore, a distinction of active and inactive TPBs becomes obsolete. 


\subsection{Distribution function of chord lengths}

The chord length of a random set $\Xi$ in direction $v$ is a random variable, defined as the length of a typical line segment in $\Xi \cap \ell$, where $\ell$ is the line with direction $v$ containing the origin [24]. Comparing the distribution function of chord lengths in different directions allows a quantification of anisotropy effects in stationary random sets, see Section 4.1. Note that in case of an isotropic stationary random set, the distribution function of chord lengths does not depend on the choice of $v$.

\subsection{Specific area of interfaces}

The specific area of an interface is the surface area per unit volume between two phases, e.g. between pores and $\mathrm{Ni}$. The specific area between phases $\Xi_{i}$ and $\Xi_{j}$, where $i, j \in\{1,2,3\}$ and $i \neq j$ is defined by

$$
I_{i, j}=\frac{1}{\nu_{3}(W)} \mathbb{E} \mathcal{H}_{2}\left(\Xi_{i} \cap \Xi_{j} \cap W\right),
$$

where $\mathcal{H}_{2}$ denotes the two-dimensional Hausdorff-measure in $\mathbb{R}^{3}$, i.e. the area of a two-dimensional object in 3D. Note that $I_{i, j}$ does not depend on the specific choice of the observation window $W$. To estimate $I_{i, j}$ we first determine all voxels at the boundary between $\Xi_{i}$ and $\Xi_{j}$. The area of the phase boundary is then estimated by a weighted sum of the boundary voxels as it is described in [24].

\section{Stochastic model}

In this section we present the parametric stochastic microstructure model for the description and simulation of Ni-YSZ anodes. We start with a simple model which is able to describe three-phase materials where all three phases are completely connected with probability 1 for a certain constellation of model parameters and the volume fractions can be easily expressed by the model parameters. The corresponding formula is derived by a simulation study. However, this model is not yet flexible enough to describe the given experimental image data with respect to constrictivity. At the end of this section a generalization of the model is proposed, which solves this problem. The generalized model is finally fitted to experimental image data in Section 4.

The model is based on the following idea, which is visualized in Figure 4. To begin with we consider three random point patterns in $\mathbb{R}^{3}$, where each 

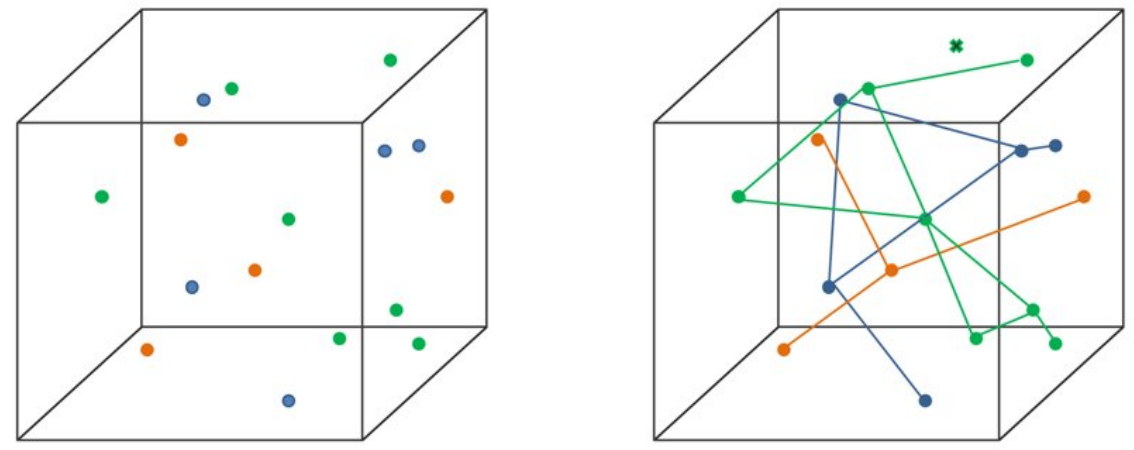

Figure 4: Modeling idea for generating three-phase microstructures: Starting with three random point patterns (left), three random graphs are constructed (right). Finally each point in the observation window is attached to the phase the graph of which is closest to the point, e.g., the point marked by a cross is attached to the phase corresponding to the green graph.

of them is the vertex set of a random geometric graph, which is modeled in a second step. The three random point patterns are modeled by three independent homogeneous Poisson point processes in $\mathbb{R}^{3}$, see [17]. This means that the points of each vertex set are located completely at random in the

5 three-dimensional space with a given intensity. Note that the intensity of a homogeneous Poisson point process is the expected number of points in the unit cube $[0,1]^{3}$.

For modeling the edges of the random geometric graphs we use so-called beta-skeletons, since for certain parameter constellations these graphs are completely connected with probability 1 . Then, we have three model graphs, i.e. one for each phase. In the last step each point $x \in \mathbb{R}^{3}$ is assigned to the phase the corresponding graph of which is closest to $x$. In the image on the right-hand side of Figure 4 , the point $x \in \mathbb{R}^{3}$, labeled by a green cross, is attached to the phase corresponding to the green graph.

\subsection{Beta-skeletons}

For a given set of vertices in the Euclidean space, the beta-skeleton introduced in [18] defines a rule for putting edges depending on a parameter $b \geq 1$. This parameter controls the number of edges in the graph. In the present paper, we use beta-skeletons for modeling the random geometric graphs, which build the backbone of the three phases. Furthermore, we derive a formula 
for the expected total edge length of the beta-skeleton on a Poisson point process, which is strongly correlated with the volume fractions of phases in the stochastic model, see Section 3.2.2. Note that this result holds for an arbitrary dimension $d \in \mathbb{N}$.

${ }_{5} \quad$ Let $d \in \mathbb{N}, b \geq 1$ and $\varphi$ be an arbitrary (locally finite) set of vertices in $\mathbb{R}^{d}$. Then, the edge set $E_{b}$ of the beta-skeleton on $\varphi$ with parameter $b$, denoted by $G_{b}(\varphi)=\left(\varphi, E_{b}\right)$, is defined as follows. Let $x, y \in \varphi$. Denote

$$
m_{x, y}^{(1)}=\frac{b}{2} x+\left(1-\frac{b}{2}\right) y, m_{x, y}^{(2)}=\frac{b}{2} y+\left(1-\frac{b}{2}\right) x
$$

and

$$
A_{b}(x, y)=B\left(m_{x, y}^{(1)},\left|m_{x, y}^{(1)}-y\right|\right) \cap B\left(m_{x, y}^{(2)},\left|m_{x, y}^{(2)}-x\right|\right),
$$

where $B(x, r)$ denotes the open sphere centered at $x$ with radius $r>0$. Then,

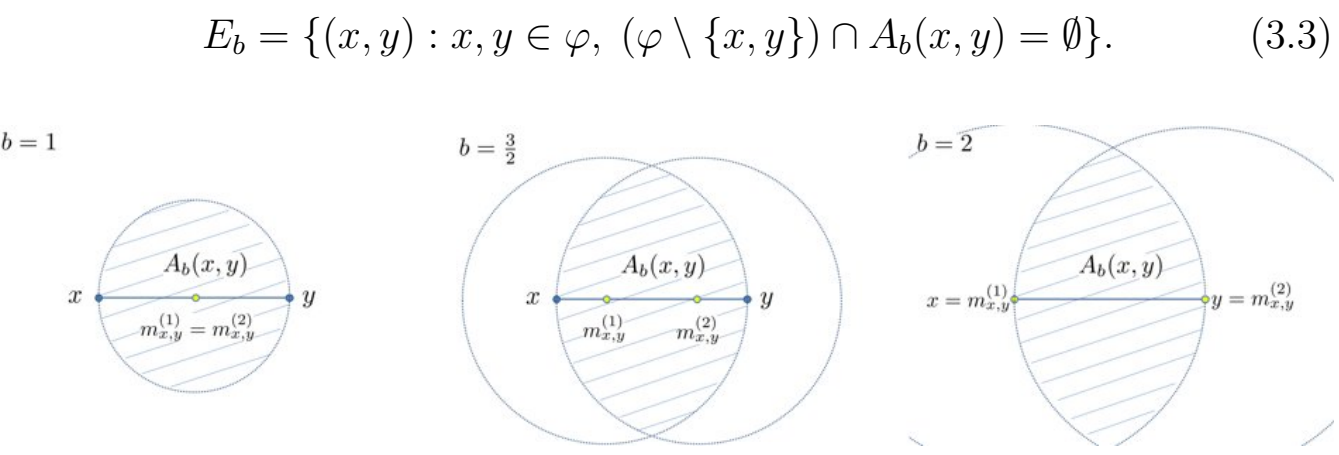

Figure 5: Critical region $A_{b}(x, y)$ for $b \in\{1,3 / 2,2\}$.

This means that for every pair of distinct vertices $x, y \in \varphi$ two spheres are defined where the midpoints $m_{x, y}^{(1)}, m_{x, y}^{(2)}$ and the radii $\left|m_{x, y}^{(1)}-y\right|,\left|m_{x, y}^{(2)}-x\right|$ of the spheres depend on the choice of the parameter $b$. The intersection of these two spheres defines the critical region $A_{b}(x, y)$. Then, $x$ and $y$ are connected by an edge in $G_{b}(\varphi)$ if there is no third point $z \in \varphi \backslash\{x, y\}$ with $z \in A_{b}(x, y)$. For $d=2$ the critical region $A_{b}(x, y)$ is visualized for different parameters $b$ in Figure 5.

It is clear that for increasing $b$ the critical region $A_{b}(x, y)$ is increasing. Thus for a given set of vertices $\varphi$ the number of edges per unit volume is monotonously decreasing with increasing $b$. Consequently the beta-skeleton on $\varphi$ is completely connected for all $1 \leq b \leq 2$ if it is completely connected for $b=2$. In case of $b=2$ the beta-skeleton coincides with the so-called 
relative neighborhood graph [18]. Since the relative neighborhood graph is completely connected with probability 1 , see [13], if the vertex set is given by a homogeneous Poisson point process, the beta-skeleton on a Poisson point process is completely connected with probability 1 for $1 \leq b \leq 2$.

5 Besides these qualitative properties of the beta-skeleton it is also possible to derive a formula for the expected total edge length of the beta-skeleton on a homogeneous Poisson point process in the unit cube $[0,1]^{d}$. In [1] a formula for the expected total edge length for a wider class of random geometric graphs, where the vertices are generated by a Poisson point process, was given for $d=2$. In the microstructure model presented here, the result for $d=3$ is used to predict the volume fractions of pores, YSZ and Ni by the aid of model parameters, see Section 3.2. To formulate the following result we denote the line segment between points $x, y \in \varphi$ by $[x, y]$ and we write $\sum_{x, y \in \varphi}^{\neq}$for the sum over all pairs of distinct points $x, y \in \varphi$.

Proposition 1. Let $d \in \mathbb{N}, 1 \leq b \leq 2$ and $X$ be a homogeneous Poisson point process in $\mathbb{R}^{d}$ with intensity $\lambda>0$. Let $G_{b}(X)=\left(X, E_{b}\right)$ be the beta-skeleton on $X$ with parameter $b$. Then, the expected total edge length

$$
e_{\lambda, b}=\frac{1}{2} \mathbb{E} \sum_{x, y \in X}^{\neq} \mathcal{H}_{1}\left([x, y] \cap[0,1]^{d}\right)
$$

of $G_{b}(X)$ in $[0,1]^{d}$ is given by

$$
e_{\lambda, b}=\frac{2^{d-1+\frac{1}{d}} \lambda^{1-\frac{1}{d}} \pi^{\frac{1}{2 d}}}{b^{d+1} d\left(\int_{0}^{\arccos \left(1-\frac{1}{b}\right)} \sin ^{d}(t) \mathrm{d} t\right)^{1+\frac{1}{d}}} \frac{\left(\Gamma\left(\frac{d+1}{2}\right)\right)^{1+\frac{1}{d}} \Gamma\left(\frac{1}{d}\right)}{\Gamma\left(\frac{d}{2}+1\right)},
$$

where $\Gamma:[0, \infty) \longrightarrow[0, \infty)$ denotes the gamma function. For $d=3$ this term simplifies to

$$
e_{\lambda, b}=8 \Gamma\left(\frac{4}{3}\right) \sqrt[3]{\frac{12 \lambda^{2}}{\pi(3 b-1)^{4}}}
$$

A proof of Proposition 1 is given in the Appendix.

\subsection{Three-phase microstructure model}

\subsubsection{Model description}

The three-phase microstructure model is defined by means of three betaskeletons. Let $X_{1}, X_{2}, X_{3}$ be independent homogeneous Poisson point processes with intensities $\lambda_{1}, \lambda_{2}, \lambda_{3}>0$. Let $b_{1}, b_{2}, b_{3} \geq 1$ and define $G_{i}=G_{b_{i}}\left(X_{i}\right)$ 
for each $i \in\{1,2,3\}$, i.e., $G_{i}$ is the beta-skeleton with parameter $b_{i}$ and vertex set $X_{i}$.

Then, the three phases are defined by random sets $\Xi_{i}, i=1,2,3$, such that

$$
x \in \Xi_{i} \text { iff } d\left(x, G_{i}\right) \leq \min _{j \in\{1,2,3\}} d\left(x, G_{j}\right)
$$

5 where $d(x, G)=\min _{e \in E} \min _{y \in e}|x-y|$ is the minimum Euclidean distance from $x$ to the graph $G=(X, E)$. Note that the union of all edges in $G_{i}$ is contained in $\Xi_{i}$ by definition. Since $G_{i}$ is completely connected if $1 \leq b_{i} \leq 2$, it holds that, in this case $\Xi_{i}$ is completely connected for each $i \in\{1,2,3\}$.
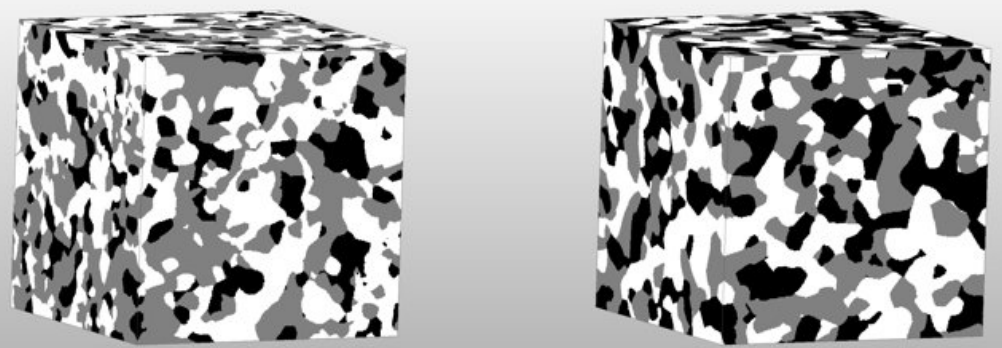

Figure 6: Realizations of the three-phase microstructure model with $W=[0,300]^{3}$ and the following parameter constellations: Left: $\lambda_{1}=\lambda_{2}=\lambda_{3}=2 \cdot 10^{-5}, b_{1}=b_{2}=b_{3}=1.5$. Right: $\lambda_{1}=3 \cdot 10^{-5}, \lambda_{2}=\lambda_{3}=2 \cdot 10^{-5}, b_{1}=2, b_{2}=b_{3}=1$. The phases $\Xi_{1}, \Xi_{2}, \Xi_{3}$ are represented in black, dark gray and light gray, respectively.

In order to estimate the microstructure characteristics described in Section 2 the three-phase microstructure model is simulated on a voxel grid in a rectangular cuboid $W$. Then, the microstructure characteristics are estimated on the basis of these simulations. To simulate $\Xi_{1}, \Xi_{2}, \Xi_{3}$ we proceed in four steps. At first the three independent Poisson point processes are simulated [21]. In a second step the beta-skeletons are computed by checking for each pair of points if they are connected by an edge of the corresponding betaskeleton. Then the three beta-skeletons are discretized on the voxel grid. In the last step the algorithm given in [9] is used to compute the distance from each voxel $x$ to each of the three discretized beta-skeletons. Thus we can determine the phase which $x$ belongs to. Two realizations of the microstructure model obtained in this way in $W=[0,300]^{3}$ are visualized in Figure 6. 
In order to avoid edge effects we apply a so-called plus-sampling, i.e., we simulate the virtual microstructures on a larger domain $W^{\prime}$ with $W \subset W^{\prime}$ and estimate the microstructure characteristics on $W$.

Based on simulations of the three-phase microstructure model, we discuss 5 the influence of model parameters on volume fractions, specific areas of interfaces and constrictivities of the three phases. While it is possible to obtain arbitrary constellations of volume fractions and predicting them on the basis of model parameters, the model is not flexible enough to reproduce a wide range of constrictivities for given volume fractions.

\subsubsection{Volume fraction}

Since each phase of the three-phase microstructure model is given by all those points which are closer to the graph of the considered phase than to the other two graphs, a correlation between the total edge lengths of the three graphs and the (empirical) volume fraction of the corresponding phases is intuitively expected. We analyze this correlation on the basis of simulations in $W=[0,500]^{3}$ with a plus-sampling on $W^{\prime}=[-50,550]^{3}$. Here we simulate one realization for each parameter constellation of the set

$$
\begin{aligned}
& \left\{\left(\lambda_{1}, \lambda_{2}, \lambda_{3}, b_{1}, b_{2}, b_{3}\right) \in\left\{9.26 \cdot 10^{-7}, 1.85 \cdot 10^{-6}, 2.78 \cdot 10^{-6}\right\}^{3}\right. \\
& \left.\quad \times\{1,3 / 2,2\}^{3}: \lambda_{1} \geq \lambda_{2} \geq \lambda_{3}\right\}
\end{aligned}
$$

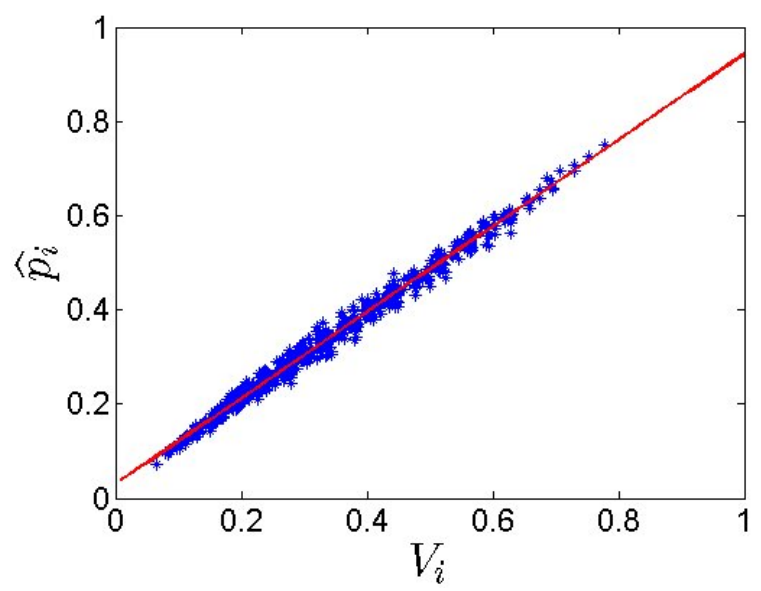

Figure 7: The volume fractions of phases can be predicted by a function of the parameters $\lambda_{1}, \lambda_{2}, \lambda_{3}, b_{1}, b_{2}, b_{3}$ using a linear regression model. 
We describe the relationship between (empirical) volume fractions and the values

$$
V_{i}=\frac{e_{\lambda_{i}, b_{i}}}{e_{\lambda_{1}, b_{1}}+e_{\lambda_{2}, b_{2}}+e_{\lambda_{3}, b_{3}}}
$$

by the following linear regression model

$$
\widehat{p}_{i}=0.9132 V_{i}+0.0292+\varepsilon_{1, i}
$$

for each $i \in\{1,2,3\}$, where $\varepsilon_{1, i}$ is normally distributed with mean 0 and 5 standard deviation 0.013 , i.e. $\varepsilon_{1, i} \sim \mathrm{N}\left(0,0.013^{2}\right)$. Besides the good visual fit, as shown in Figure 7, the coefficient of determination $R^{2}=0.99$ indicates a high predictability of the volume fractions by the total edge lengths and therefore, in view of Equation (3.6), by the model parameters. In order to validate the obtained regression model, 100 further realizations have been simulated where the parameters were randomly chosen. This means that all parameters are independently and uniformly distributed in certain intervals, to be more precise $\lambda_{i} \sim \mathrm{U}\left(9.26 \cdot 10^{-7}, 2.78 \cdot 10^{-6}\right)$ and $b_{i} \sim \mathrm{U}([1,2])$ for each $i \in\{1,2,3\}$. The errors $\widehat{p}_{i}-0.9132 V_{i}-0.0292$ obtained by simulation have (empirical) mean and (empirical) standard deviation of 0.01 and 0.017 , 15 respectively, which shows that the linear regression model fits the relationship between volume fractions and expected total edge lengths very well.

The derived relationship between expected total edge lengths and volume fractions allows us to reduce the vector of (free) model parameters $\left(\lambda_{1}, \lambda_{2}, \lambda_{3}, b_{1}, b_{2}, b_{3}\right)$ under the condition that the volume fractions $p_{1}, p_{2}$ and $p_{3}$ are fixed. Namely using Equations (3.6) and (3.10), for given volume fractions and given model parameters $\lambda_{1}, b_{1}, b_{2}, b_{3}$, we get the approximation

$$
\lambda_{i} \approx \lambda_{1}\left(\frac{p_{i}}{p_{1}}\right)^{3 / 2}\left(\frac{3 b_{i}-1}{3 b_{1}-1}\right)^{2}
$$

for each $i \in\{2,3\}$. This approximation can be improved by solving Equation (3.10) for $\lambda_{2}$ and $\lambda_{3}$.

\subsubsection{Specific area of interfaces}

Besides the relationship derived in Section 3.2.2 between model parameters and volume fractions of the three phases there is a strong correlation between volume fractions and specific areas of interfaces. Here an analogy to very simple three-phase models, so-called independent tiling models can be drawn. 


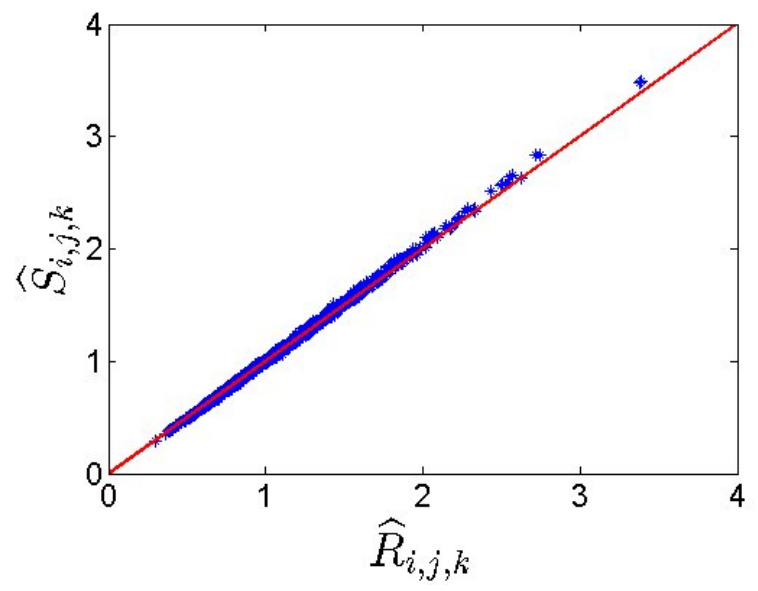

Figure 8: Relationship between the expressions $\left(I_{i, j}+I_{i, k}\right) /\left(I_{i, j}+I_{j, k}\right)$ and $\left(p_{i}\left(p_{j}+\right.\right.$ $\left.\left.p_{k}\right)\right) /\left(p_{j}\left(p_{i}+p_{k}\right)\right)$ estimated from simulated three-phase microstructures. The line through the origin with slope 1 is drawn in red.

Consider the following regular tilings of space: A square grid in $2 \mathrm{D}$, a hexagonal grid in 2D, and a cubic grid in 3D. Then, the corresponding independent tiling model of such grids is defined in the following way: The origin is located at random in one of the cells of the considered grid and each cell is 5 independently assigned to $\Xi_{i}$ with probability $p_{i}$ for each $i \in\{1,2,3\}$, where $p_{1}, p_{2}, p_{3} \geq 0$ and $p_{1}+p_{2}+p_{3}=1$. For these three independent tiling models it is not difficult to show that the following relationship

$$
\frac{I_{i, j}+I_{i, k}}{I_{i, j}+I_{j, k}}=\frac{p_{i}\left(p_{j}+p_{k}\right)}{p_{j}\left(p_{i}+p_{k}\right)}
$$

holds. We denote the left-hand side by $S_{i, j, k}=\left(I_{i, j}+I_{i, k}\right) /\left(I_{i, j}+I_{j, k}\right)$ and the right-hand side by $R_{i, j, k}=\left(p_{i}\left(p_{j}+p_{k}\right)\right) /\left(p_{j}\left(p_{i}+p_{k}\right)\right)$ for abbreviation.

Simulating 3D realizations with our microstructure model for the same parameter set as in Section 3.2.2, we can estimate $S_{i, j, k}$ and $R_{i, j, k}$. The corresponding estimators are denoted by $\widehat{S}_{i, j, k}=\left(\widehat{I}_{i, j}+\widehat{I}_{i, k}\right) /\left(\widehat{I}_{i, j}+\widehat{I}_{j, k}\right)$ and $\widehat{R}_{i, j, k}=\left(\widehat{p}_{i}\left(\widehat{p}_{j}+\widehat{p}_{k}\right)\right) /\left(\widehat{p}_{j}\left(\widehat{p}_{i}+\widehat{p}_{k}\right)\right)$. Figure 8 shows that the developed microstructure model behaves rather similar as the independent tiling model with respect to the specific areas of interfaces. The reason for this analogy might be the strong independence property between the three graphs. We come back to this analogy in Section 4.4. 


\subsubsection{Constrictivity}

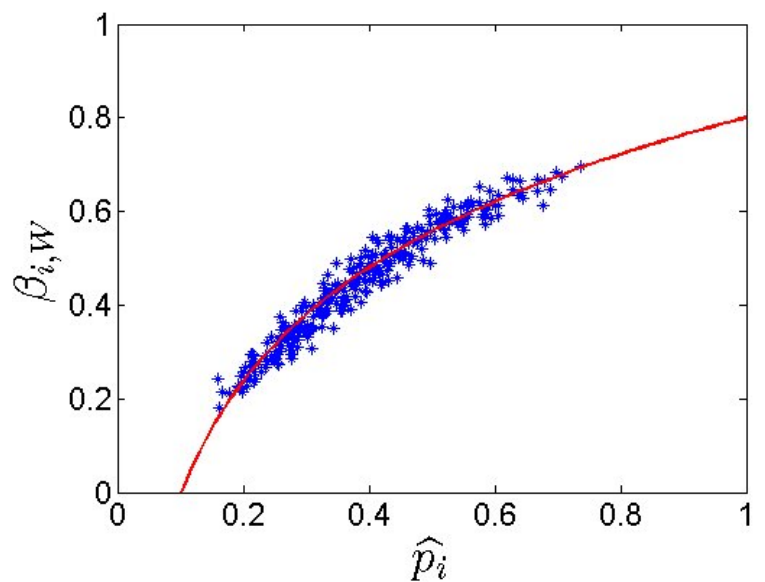

Figure 9: Relationship between constrictivity $\beta_{i, W}$ and $p_{i}$ estimated from simulated threephase microstructures. The model introduced in Section 3.2.1 is not able to generate constrictivities in a sufficiently wide range for a given volume fraction. The red line shows the fit by linear regression between $\log \widehat{p}_{i}$ and $\widehat{\beta}_{i, W}$.

For the same realizations of our microstructure model that were used in Section 3.2.2 for the investigation of the relationship between model parameters and volume fractions, the constrictivities $\beta_{1, W}, \beta_{2, W}, \beta_{3, W}$ with $W=$ ${ }_{5}[0,500]^{3}$ have been estimated. The results are visualized in Figure 9. One can observe that the model introduced in Section 3.2.1 is not flexible enough with respect to constrictivity since there is a strong correlation between volume fraction and constrictivity of the same phase. This correlation can be modeled by

$$
\widehat{\beta}_{i, W}=0.35 \log \widehat{p}_{i}+0.8+\varepsilon_{2, i}
$$

with $\varepsilon_{2, i} \sim N\left(0,0.028^{2}\right)$. The coefficient of determination is 0.95 .

This absence of flexibility regarding constrictivity can be explained by the fact that each phase is in some sense homogeneously located around the corresponding graph. Homogeneity means here that there are no regions of the graph which tend to attract the corresponding phase more than others. Thus there are less bottlenecks in the phases than this is required for a systematic variation of volume fractions and constrictivities in virtual microstructures. This problem will be solved by a generalization of the model in Section 3.3. Furthermore, in the context of the generalized model, we will 
describe the estimation of all model parameters in order to achieve a good fit to experimental image data of three-phase microstructures in Section 4.

Nevertheless, we have created a simple parametric stochastic microstructure model for three phase materials where it is possible to choose the param-

5 eters in such a way that all phases are completely connected with probability 1 and the volume fractions of phases can be predicted by the aid of model parameters. Note that the model can be easily simulated without any kind of iterative procedure.

\subsection{Generalization of the model}

The generalized three-phase microstructure model is defined in the same way as the model presented in Section 3.2, but when we decide to which phase an arbitrary point $x \in W$ belongs, we do not use the Euclidean distance anymore. Instead, we use a parametric distance such that by variation of the parameter the corresponding phase is more or less accumulated around the vertices of the graph. The generalized model is formally defined as follows.

Let $\gamma_{1}, \gamma_{2}, \gamma_{3} \geq 1$ and let $G_{1}, G_{2}, G_{3}$ be the beta-skeletons introduced in Section 3.2. The three phases are now defined by random sets $\Xi_{i}, i=1,2,3$, such that

$$
x \in \Xi_{i} \text { iff } d_{\gamma_{i}}^{\prime}\left(x, G_{i}\right) \leq \min _{j \in\{1,2,3\}} d_{\gamma_{j}}^{\prime}\left(x, G_{j}\right)
$$

where

$$
d_{\gamma_{i}}^{\prime}\left(x, G_{i}\right)=\min \left\{\gamma_{i} d\left(x, G_{i}\right), d\left(x, X_{i}\right)\right\} .
$$

Here $d\left(x, X_{i}\right)=\min _{y \in X_{i}}|x-y|$ is the minimum Euclidean distance from $x$ to the set of vertices $X_{i}$.

The new distance measure $d_{\gamma}^{\prime}$ fulfills $d_{\gamma}^{\prime}(x, G)=0$ for each $x$ located on the edge set of the graph $G$ and for each admissible $\gamma$. Thus in the generalized model it is still valid that all three phases are completely connected with probability 1 in case that $1 \leq b_{i} \leq 2$ for each $i \in\{1,2,3\}$. If $\gamma=1$ it holds that $d_{\gamma}^{\prime}(x, G)=d(x, G)$, i.e. $d_{\gamma}^{\prime}(x, G)$ coincides with the Euclidean distance from $x$ to $G$. With increasing $\gamma$ the distance from $x$ to the graph increases. However, this increase becomes smaller the closer $x$ is to the nearest vertex of the graph $G$ as shown in Figure 10.

30 Figure 11 shows the development of constrictivity in case that the parameters $\lambda_{1}, \lambda_{2}, \lambda_{3}, b_{1}, b_{2}, b_{3}$ are fixed, where $\lambda_{1}=\lambda_{2}=\lambda_{3}=1.25 \cdot 10^{-5}, b_{1}=b_{2}=$ $b_{3}=1.5$ and $\gamma_{1}=\gamma_{2}=\gamma_{3}=\gamma$ where $\gamma$ is varied between 1 and 10 . Note that in this case it holds that $p_{1}=p_{2}=p_{3}=1 / 3$. One can observe that with 

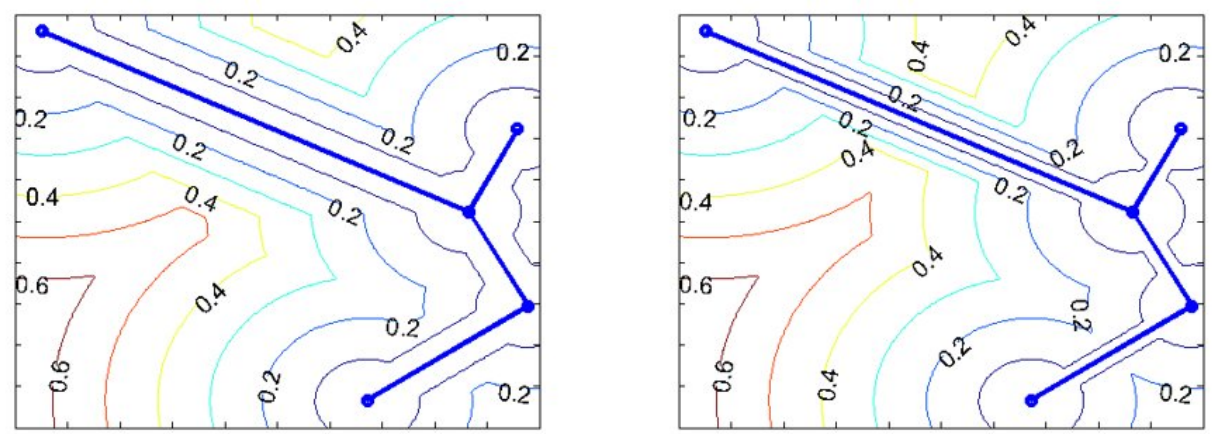

Figure 10: Contour lines of the distance to a given graph represented in blue with respect to $d_{\gamma}^{\prime}$ for $\gamma=2$ (left) and $\gamma=4$ (right).

increasing $\gamma$, constrictivity decreases for $\gamma \geq 2$. This is not surprising due to the definition of $d_{\gamma}^{\prime}$ wherein each phase is more accumulated around the vertices with increasing $\gamma$ while the volume fraction does not change. This leads to the occurrence of bottlenecks and thus to decreasing constrictivities.

${ }_{5}$ The small increase of constrictivity for $\gamma<2$ is due to a faster decrease of $r_{\max }$ than $r_{\min }$ for small $\gamma$.

Finally, in a last step, the model is further generalized. The boundary of the three phases is smoothed in order to have the possibility to decrease the length of TPB. For this purpose we define a kind of Gaussian smoothing for three-phase microstructures. At first we smooth the boundary between pores and the union of $\mathrm{Ni}$ and YSZ phases by a Gaussian smoothing. In a second step the boundary between $\mathrm{Ni}$ and YSZ phases is also smoothed by a Gaussian smoothing.

We consider a function $F$ on $\mathbb{R}^{3}$, which allocates a real number to each voxel according to the phase $x$ belongs to. Furthermore, let $\theta>0$ and consider the function

$$
\varphi_{F}(x)=\frac{\int_{\mathbb{R}^{3}} F(y) \cdot \exp \left(-\frac{|x-y|^{2}}{2 \theta^{2}}\right) \mathrm{d} y}{\int_{\mathbb{R}^{3}} \exp \left(-\frac{|x-y|^{2}}{2 \theta^{2}}\right) \mathrm{d} y}
$$

for each $x \in \mathbb{R}^{3} \cap W$. In particular, we choose the function $F_{1}$ on $\mathbb{R}^{3}$ as $F_{1}(x)=1$ if $x \in \Xi_{1}$ and $F_{1}(x)=2$ otherwise. Moreover, we define the function $F_{2}$ on $\mathbb{R}^{3}$ by $F_{2}(x)=3 / 2$ if $x \in \Xi_{1}, F_{2}(x)=2$ if $x \in \Xi_{2}$ and $F_{2}(x)=1$ otherwise. Then, the phases of the smoothed microstructure, denoted by 


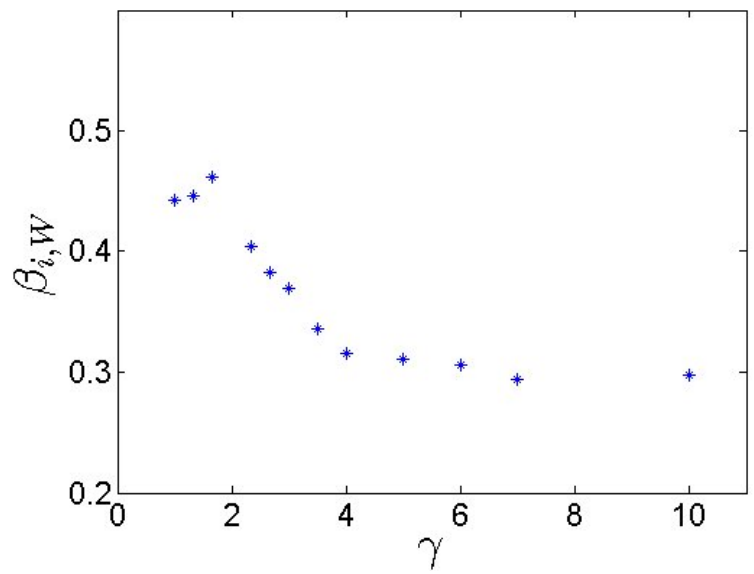

Figure 11: Constrictivity $\widehat{\beta}_{i}$ over $\gamma$ estimated for the three-phase microstructure model with parameters $\lambda_{1}=\lambda_{2}=\lambda_{3}=1.25 \cdot 10^{-5}, b_{1}=b_{2}=b_{3}=1.5, \gamma_{1}=\gamma_{2}=\gamma_{3}=\gamma$. For each parameter set, 9 realizations on $W=[0,500]^{3}$ are simulated.

$\Xi_{1}^{\prime}, \Xi_{2}^{\prime}, \Xi_{3}^{\prime}$, are given by $\Xi_{1}^{\prime}=\left\{x \in \mathbb{R}^{3}: \varphi_{F_{1}}(x) \leq 1\right\}, \Xi_{2}^{\prime}=\left\{x \in \mathbb{R}^{3}\right.$ : $\left.\varphi_{F_{1}}(x) \geq 1, \varphi_{F_{2}}(x) \leq 3 / 2\right\}$ and $\Xi_{3}^{\prime}=\left\{x \in \mathbb{R}^{3}: \varphi_{F_{1}}(x) \geq 1, \varphi_{F_{2}}(x) \geq 3 / 2\right\}$.

For simulation purposes, the smoothing is applied on the discretized microstructure. Thus to compute $\varphi_{F_{1}}$ and $\varphi_{F_{2}}$, we approximate the integrals in the definition of $\varphi_{F}$ by sums, i.e., $\varphi_{F}$ is approximated by $\widehat{\varphi}_{F}$ defined by

$$
\widehat{\varphi}_{F}(x)=\frac{\sum_{y \in \mathcal{V}} F(y) \cdot \exp \left(-\frac{|x-y|^{2}}{2 \theta^{2}}\right)}{\sum_{y \in \mathcal{V}} \exp \left(-\frac{|x-y|^{2}}{2 \theta^{2}}\right)},
$$

for each $x \in \mathcal{V}$, where $\mathcal{V}$ denotes the set of all voxels in $W$.

After the application of smoothing the complete connectivity of the three phases can not be guaranteed in general. However, simulations show that for small values of the smoothing parameter $\theta$ the connectivity of the phases is still very good, see Table 2 in Section 4.1.

Note that the three-phase microstructure model originally introduced in Section 3.2.1 has been generalized such that the number of parameters is increased from 6 to 10. By this generalization a better control over constrictivity of the phases and over the length of TPB is achieved. 


\section{Model fitting and validation}

\subsection{Image data of $N i$-YSZ anodes}

Having defined the generalized three-phase microstructure model we show that this model is able to describe complex 3D experimental image data repre-

5 senting real Ni-YSZ anodes. We fit our model to the fine Ni-YSZ microstructure in SOFC anodes before degradation which was recently described and analyzed in [25]. The data obtained by FIB-tomography is visualized in Figure 12. The data represents a Ni-YSZ anode on a rectangular cuboid $W=[0,20 \mu \mathrm{m}] \times[0,25 \mu \mathrm{m}] \times[0,15 \mu \mathrm{m}]$ with a resolution of $30 \mathrm{~nm}$.
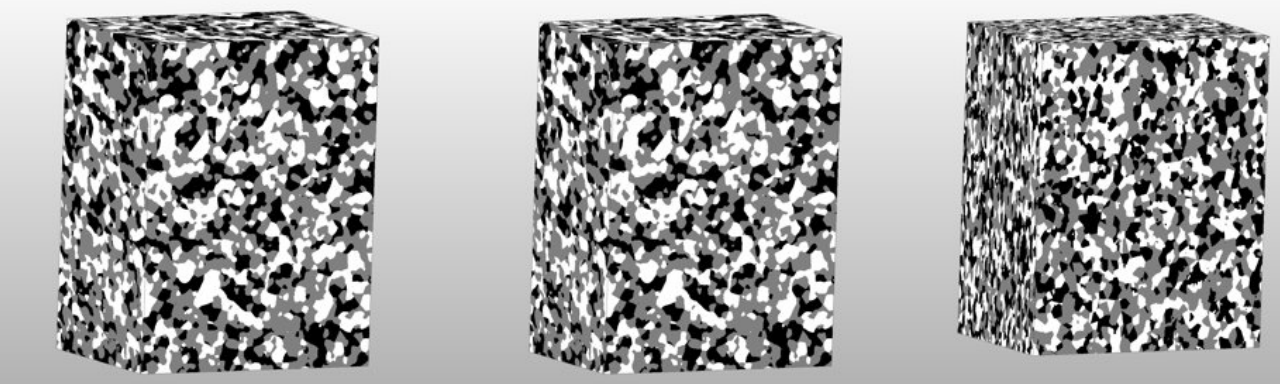

Figure 12: Left: 3D experimental image data of Ni-YSZ anodes. Pores are represented in black, the YSZ phase in dark gray and the Ni phase in light gray. Center: Scaled image data. Right: Simulated microstructure with fitted parameters.

In experimental image data anisotropy effects with respect to chord-length distributions can be observed in Figure 13. For all three phases and for all lengths greater than $1 \mu \mathrm{m}$, the estimated distribution functions of chord lengths in $z$ - direction is smaller than the distribution functions in $x$ - and $y$ - direction. This indicates that all three phases are elongated in $z$ - direction.

Since the considered material is not expected to have such anisotropy, these effects in experimental image data are ascribed to FIB-imaging. Thus we scale the image data in order to obtain an isotropic structure. Note that a scaling of the image data influences TPB since the scaling changes the voxel size. However, different kinds of scaling have a different influence on TPB. In case that scaling is combined with a smoothing of the image the TPB decreases. 

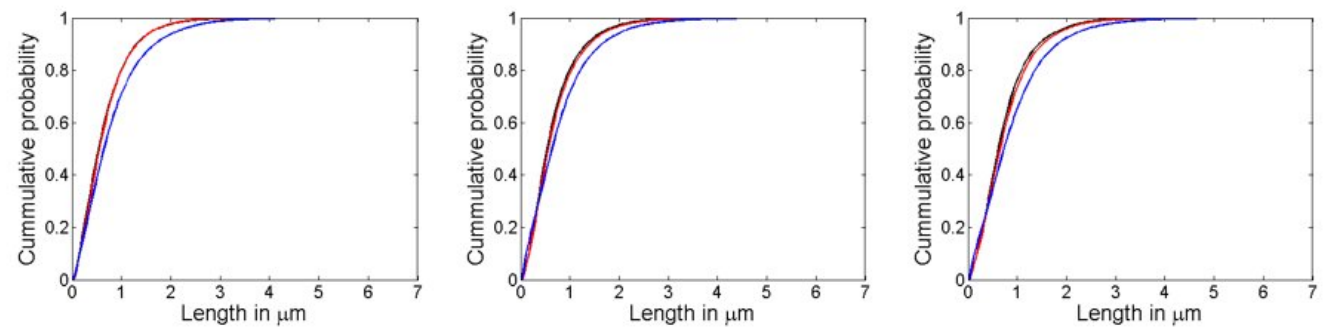

Figure 13: Distribution functions of chord lengths with respect to the three main directions estimated from experimental image data. The black (red, blue) curve represents the distribution functions of chord lengths in $x-(y-, z-)$ direction. From left to right: Distribution functions of chord lengths for pores, YSZ and Ni.

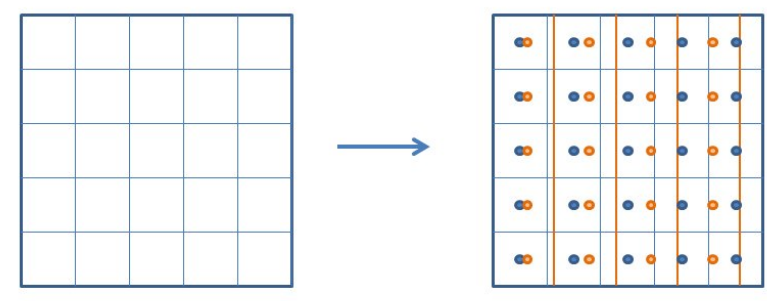

Figure 14: Nearest-neighbor interpolation in 2D. The original voxel grid is represented in blue, the transformed voxel grid in orange. The centers of voxels are represented by small disks in the corresponding colour.

For the purpose of scaling we use the so-called nearest-neighbor interpolation visualized in Figure 14. It turns out that a scale of the $z$-direction by 0.88 leads to the best result with respect to the accordance of distribution functions of chord lengths in the three main directions. After scaling 5 we obtain a new voxel grid and we have to determine the phase of each of these new voxels. For this purpose, for each center $c$ of a new voxel $v$ we consider the voxel $w$ in the original grid the center of which is closest to $c$. Then, the voxel $v$ belongs to the same phase as $w$. Figure 15 shows that the scaled image can be considered as isotropic with respect to chordlength distributions. The result of scaling is visualized in the center of Figure 12.

Table 1 shows the microstructure characteristics described in Section 2 for experimental image data before and after the scaling. The transport relevant characteristics are computed with respect to the transport directions in the Ni-YSZ anode. It can be observed that the characteristics are hardly changed 

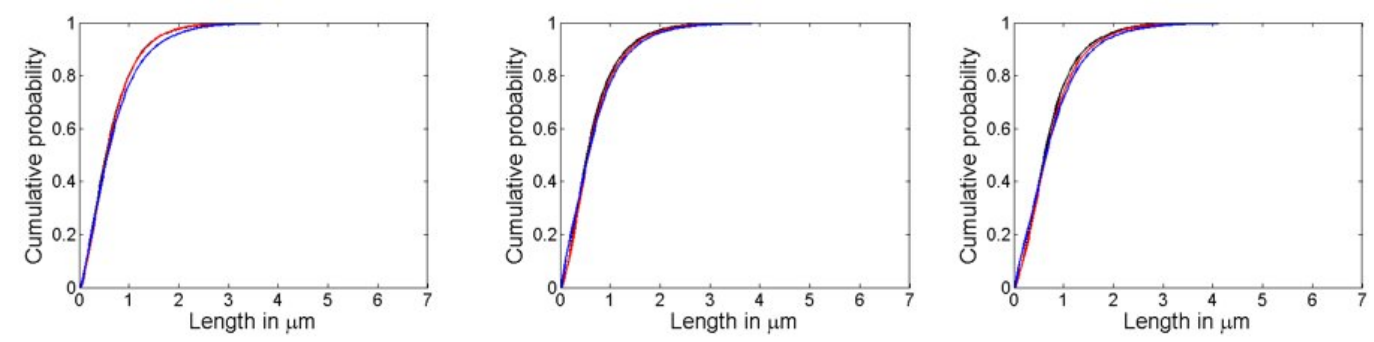

Figure 15: Distribution functions of chord lengths in the three main directions estimated from transformed image data. The black (red, blue) curve represents the distribution functions of chord lengths in $x-(y-, z-)$ direction. From left to right: Distribution functions of chord lengths for pores, YSZ phase and Ni phase.

\begin{tabular}{ccccccccccc} 
& $\widehat{p}_{1}$ & $\widehat{p}_{2}$ & $\widehat{p}_{3}$ & $\widehat{\tau}_{1}$ & $\widehat{\tau}_{2}$ & $\widehat{\tau}_{3}$ & $\widehat{\beta}_{1}$ & $\widehat{\beta}_{2}$ & $\widehat{\beta}_{3}$ & $\widehat{\delta}_{1}$ \\
\hline Tomo. & 0.26 & 0.42 & 0.32 & 1.28 & 1.11 & 1.18 & 0.30 & 0.41 & 0.29 & 0.0033 \\
\hline Tomo. scaled & 0.25 & 0.42 & 0.33 & 1.26 & 1.10 & 1.17 & 0.31 & 0.42 & 0.33 & 0.0036 \\
\hline Simulation & 0.28 & 0.40 & 0.32 & 1.17 & 1.10 & 1.13 & 0.24 & 0.44 & 0.33 & 0.0035
\end{tabular}

Table 1: Comparison of microstructure characteristics for scaled tomography data, tomography data and simulated data. The different phases are numbered in the following way: 1 - Pores, 2 - YSZ, 3 - Ni.

by the scaling.

In experimental image data it can be seen that the three phases are almost completely connected. As a measure for connectivity we compute the fraction $c_{i, x}\left(c_{i, y}, c_{i, z}\right)$ of each phase $i \in\{1,2,3\}$ that is connected in $x-(y-, z-)$ direction. The results are given in Table 2. All values are close to one, which indicates a high connectivity. Thus it is appropriate to model the given experimental image data by a stochastic model that reproduces these connectivity properties.

\begin{tabular}{cccccccccc} 
& $c_{1, x}$ & $c_{1, y}$ & $c_{1, z}$ & $c_{2, x}$ & $c_{2, y}$ & $c_{2, z}$ & $c_{3, x}$ & $c_{3, y}$ & $c_{3, z}$ \\
\hline Tomo. & 0.987 & 0.985 & 0.987 & 1.000 & 1.000 & 1.000 & 0.986 & 0.987 & 0.986 \\
\hline Tomo. scaled & 0.988 & 0.985 & 0.987 & 0.999 & 0.999 & 0.999 & 0.986 & 0.987 & 0.986 \\
\hline Simulation & 1.000 & 1.000 & 1.000 & 0.997 & 0.997 & 0.997 & 1.000 & 1.000 & 1.000
\end{tabular}

Table 2: Comparison of connectivity for tomography data, scaled tomography data and simulated data. For each $i \in\{1,2,3\}$ the value $c_{i, x}$ denotes the fraction of the $i$-th phase that is connected in $x$-direction. The definition of $c_{i, y}$ and $c_{i, z}$ is analogous. The different phases are numbered in the following way: 1 - Pores, 2 - YSZ, 3 - Ni. 


\subsection{Fit of model parameters to experimental image data}

Moreover, in Table 1 microstructure characteristics of experimental image data and the stochastic model are compared. Note that the parameters of the stochastic model are fitted to the volume fractions, length of TPB and 5 to geodesic tortuosities as well as constrictivities of $\mathrm{Ni}$ and YSZ, i.e., we minimize the following cost function of relative errors between microstructure characteristics of simulated and scaled image data:

$$
3 \sum_{i=2}^{3} \frac{\left|\widehat{p}_{i, s i m}-p_{i}\right|}{p_{i}}+2 \sum_{i=2}^{3} \frac{\left|\widehat{\beta}_{i, s i m}-\beta_{i}\right|}{\beta_{i}}+\sum_{i=2}^{3} \frac{\left|\widehat{\tau}_{i, s i m}-\tau_{i}\right|}{\tau_{i}}+\frac{\left|\widehat{\delta}_{1, s i m}-\delta_{1}\right|}{\delta_{1}} .
$$

For this purpose we use the Nelder-Mead method introduced in [23]. This method, also called downhill-simplex method is a direct search method, i.e., it is used for non-linear optimization without using derivatives of the function that has to be minimized. Although there are not many results about convergence of this method it is successfully used in many applications. This is adequate here since we do not need to find the best, but a sufficiently good parameter constellation, which shows that the model is able to generate realistic microstructures. For more information about the Nelder-Mead method the reader is referred to [7].

\begin{tabular}{cc} 
Parameters & Fitted values \\
\hline$\lambda_{1}$ & $0.87 \mu \mathrm{m}^{-3}$ \\
\hline$\lambda_{2}$ & $1.18 \mu \mathrm{m}^{-3}$ \\
\hline$\lambda_{3}$ & $0.95 \mu \mathrm{m}^{-3}$ \\
\hline$b_{1}$ & 2.11 \\
\hline$b_{2}$ & 1.97 \\
\hline$b_{3}$ & 1.94 \\
\hline$\gamma_{1}$ & 4.47 \\
\hline$\gamma_{2}$ & 4.31 \\
\hline$\gamma_{3}$ & 4.12 \\
\hline$\theta$ & 0.69
\end{tabular}

Table 3: Parameters fitted by the Nelder-Mead method.

The microstructure characteristics for a certain parameter set are estimated on the basis of simulations on $W=[0,15 \mu \mathrm{m}]^{3}$ with voxel size $30 \mathrm{~nm}$. By the Nelder-Mead method the optimal parameter set given in Table 3 is obtained. It leads to a very good fit with respect to the microstructure characteristics that are considered in the cost function, i.e. $p_{2}, p_{3}, \beta_{2}, \beta_{3}, \tau_{2}, \tau_{3}, \delta_{1}$. 
Note that the remaining characteristics mean geodesic tortuosity $\tau_{1}$ and constrictivity $\beta_{1}$ of pores are not taken into account in the cost function. However, the microstructure characteristics of the simulated microstructures are quite similar to the ones of experimental data. Moreover, note that the fitting 5 procedure leads to $b_{1}=2.11>2$. Thus the complete connectivity of $\Xi_{1}$ is not theoretically guaranteed. However, computation of connectivity properties, see Table 2 in Section 4.1, shows that this parameter constellation does also lead to almost complete connectivity of $\Xi_{1}$. In the considered realizations the phase $\Xi_{1}$ is even completely connected. Note that the values in Table 2 concerning Ni and YSZ phases that are smaller than 1 are caused by edge effects.

\subsection{Model validation}

To validate the model introduced in Section 3.3 the ratio between effective and intrinsic conductivities denoted by $\sigma_{\text {eff }}$ and $\sigma_{0}$, respectively, of $\mathrm{Ni}$ and YSZ phases are computed using the FE-method and the length of TPB is computed by the estimator $\widehat{\delta}_{2}$ described in Section 2.4. Note that FE-simulations have been performed by the software GeoDict [11].

\begin{tabular}{cccc} 
& $\sigma_{\text {eff }} / \sigma_{0}$ for YSZ phase & $\sigma_{\text {eff }} / \sigma_{0}$ for Ni phase & $\widehat{\delta}_{2}$ \\
\hline Tomo. & 0.154 & 0.074 & $2.34 \mu m^{-2}$ \\
\hline Tomo. scaled & 0.162 & 0.078 & $2.53 \mu m^{-2}$ \\
\hline Simulation & 0.152 & 0.087 & $2.77 \mu m^{-2}$
\end{tabular}

Table 4: Comparison of effective conductivities for Ni and YSZ phases and of the estimated TPB for tomography data, scaled tomography data and simulated data.

The results are given in Table 4 . Note that the three characteristics considered in this table are not used for model fitting. Nevertheless, we have

20 a good accordance between simulated effective conductivities and lengths of TPB. Especially the effective conductivities of the YSZ phase for experimental and simulated image data are quite close to each other. The model is thus able to capture these characteristics of Ni-YSZ anodes which are most important for the performance of the cell.

\subsection{Specific area at phase boundaries - limitations of the model}

Table 5 shows limitations of the stochastic microstructure model introduced in Section 3.3 since the model is not flexible enough with respect to the specific area at the phase boundaries. 


\begin{tabular}{cccc} 
& $\widehat{I}_{1,2}$ & $\widehat{I}_{1,3}$ & $\widehat{I}_{2,3}$ \\
\hline Tomo. & $1.01 \mu m^{-1}$ & $0.30 \mu m^{-1}$ & $1.22 \mu m^{-1}$ \\
\hline Tomo. scaled & $1.05 \mu m^{-1}$ & $0.31 \mu m^{-1}$ & $1.27 \mu m^{-1}$ \\
\hline Simulation & $0.94 \mu m^{-1}$ & $0.75 \mu m^{-1}$ & $0.99 \mu m^{-1}$
\end{tabular}

Table 5: Comparison of specific areas of interfaces between pores and YSZ phase, pores and $\mathrm{Ni}$ phase, YSZ and $\mathrm{Ni}$ phases for tomography data, scaled tomography data and simulated data.

In experimental image data the estimated specific area of the interface between pores and $\mathrm{Ni}$ phase $\left(I_{1,3}\right)$ is much smaller than in simulated data. Nonetheless, the estimated specific surface areas of pores and YSZ phase $\left(I_{1,2}\right)$ and between YSZ phase and Ni phase $\left(I_{2,3}\right)$ coincide well for experimental and simulated image data.

Recall that the fit of the shape parameters $\gamma_{1}, \gamma_{2}, \gamma_{3}$ leads to very similar values, i.e. $\left(\gamma_{1}, \gamma_{2}, \gamma_{3}\right)=(4.47,4.31,4.12)$. If $\gamma_{1}=\gamma_{2}=\gamma_{3}$, we would expect that the relationship

$$
S_{i, j, k}=\frac{I_{i, j}+I_{i, k}}{I_{i, j}+I_{j, k}} \approx \frac{p_{i}\left(p_{j}+p_{k}\right)}{p_{j}\left(p_{i}+p_{k}\right)}=R_{i, j, k},
$$

discussed in Section 3.2.3 is still valid. Table 6 shows the estimated values of the left- and right-hand side for the simulated structure. Although the equality $\gamma_{1}=\gamma_{2}=\gamma_{3}$ does not hold in our case, the values of the estimators $\widehat{S}_{i, j, k}$ and $\widehat{R}_{i, j, k}$ are very close to each other.

\begin{tabular}{cccc}
$i$ & $j$ & $\widehat{S}_{i, j, k}$ & $\widehat{R}_{i, j, k}$ \\
\hline 1 & 2 & 0.88 & 0.84 \\
\hline 2 & 3 & 1.11 & 1.10 \\
\hline 3 & 1 & 1.03 & 1.08
\end{tabular}

Table 6: Comparison of $S_{i, j, k}$ and $R_{i, j, k}$ estimated from simulated data.

Summarizing it turns out that the model is limited in the way that the specific areas of interfaces between the phases are not represented well. 15 Nevertheless, the developed three-phase microstructure model matches several microstructure characteristics, in particular those which are the most important ones with respect to electric and ionic conductivity as well as the length of TPB. 


\section{Conclusion and outlook}

In the present paper a parametric stochastic microstructure model for description and simulation of three-phase Ni-YSZ anodes was developed using methods from stochastic geometry. The model is constructed such that for 5 various parameter sets all three phases are completely connected with probability 1. Furthermore, we were able to fit the parameters of our model to experimental 3D image data.

We started with a model consisting of 6 parameters, where three betaskeletons on homogeneous Poisson point processes were modeled. Each graph builds the backbone of one of the three phases. Each phase is then given by these points which are closer (with respect to the Euclidean distance) to the corresponding graph than to the other two graphs. For this model a relationship between the expected total edge length of the three beta-skeletons and the volume fraction of the phases was established by a simulation study. Thus it is possible to describe three-phase microstructures with completely connected phases and to adjust the volume fraction by the model parameters. For fitting the model to experimental image data a generalization of the model was necessary. In order to determine to which phase a certain point belongs, we consider a (modified) parametric distance to the three 20 graphs and the boundary of the three phases is smoothed according to an additional parameter. Finally we arrive at a 10-parametric model, which is fitted successfully to experimental image data with respect to the following transport relevant microstructure characteristics: Volume fractions, mean geodesic tortuosities, constrictivities of the three phases and the length of TPB. Note that the developed model can be easily simulated in short time, which is also caused by the fact that the simulation does not involve any iterative procedure.

Using the FE-method the effective conductivities in the $\mathrm{Ni}$ and YSZ phases of simulated and experimental data have been computed. It turned out that experimental and simulated data are in good accordance to each other. This shows that the realizations of the model can be considered as realistic Ni-YSZ microstructures. Moreover, we were able to reduce the complex information of the 3D images to 10 real-valued parameters, which contain the decisive information about conductivity in the Ni and YSZ phases.

35 In further work we will vary the parameters of the stochastic model in order to obtain virtual, but realistic Ni-YSZ microstructures with different transport relevant microstructure characteristics. Using the FE-method and 
simulating the anode reaction mechanism on these virtual structures we can generate a large database in order to efficiently investigate the quantitative relationship between microstructure characteristics and the anode performance. The results of such an investigation can be validated by a few ex-

5 perimental measurements of real anodes. Therefore, this approach, based on virtual materials design performed by stochastic modeling, can be used for reliable microstructure optimization with a reduced experimental effort.

Note that the presented approach can be modified in different ways for modeling three-phase microstructures. In particular one could think of a modification towards Ni-YSZ microstructures with a bimodal pore size distribution, which was observed for the data presented in [31]. For this purpose, it might be possible to use other point process models for the vertices of the graph and to incorporate dependencies between the three point processes, instead of the model generalization considered in Sectio 3.3. Moreover, another class of connected geometric graphs can be chosen instead of the beta-skeleton for modeling the edges. So the presented approach opens new possiblities in the modeling of three-phase microstructures, which can be used in future for optimization of electrode microstructures by virtual materials design.

\section{Acknowledgments}

The authors like to thank Ole Stenzel for helpful discussions. The research presented in this paper has received funding from the Swiss National Science Foundation (SNSF, Grant Nr. 407040_154047), from the German Academic Exchange Service (DAAD) as well as from the Czech Ministery of Education (Project 7AMB14DE006). All financial supports are gratefully acknowledged.

\section{Appendix A.}

Proof of Proposition 1. At first, note that $A_{b}(x, y)$ is the union of two equally sized hyperspherical caps. The height of these hyperspherical caps is given by $h=|x-y| / 2$ and the radius of the corresponding sphere is

$$
r=|(b / 2) x+(1-b / 2) y-y|=b / 2 \cdot|x-y| .
$$

Due to results from [19] it is not difficult to see that

$$
\nu_{d}\left(A_{b}(x, y)\right)=\frac{2}{\Gamma\left(\frac{d+1}{2}\right)} \pi^{\frac{d-1}{2}}\left(\frac{b}{2}|x-y|\right)^{d} \int_{0}^{\arccos \left(1-\frac{1}{b}\right)} \sin ^{d}(t) \mathrm{d} t .
$$


Now we continue with the calculation of $e_{\lambda, b}$ where we use the notation

$$
\alpha=\lambda \nu_{d}\left(A_{b}(x, y)\right) /|x-y|^{d} .
$$

By the Slivnyak-Mecke theorem, see Theorem 3.3 in [21], we obtain

$$
\begin{aligned}
e_{\lambda, b} & =\frac{\lambda^{2}}{2} \int_{\mathbb{R}^{d}} \int_{\mathbb{R}^{d}} P\left(X \cap A_{b}(x, y)=\emptyset\right) \mathcal{H}_{1}\left([x, y] \cap[0,1]^{d}\right) \mathrm{d} x \mathrm{~d} y, \\
& =\frac{\lambda^{2}}{2} \int_{\mathbb{R}^{d}} \int_{\mathbb{R}^{d}} \int_{0}^{1} \mathbb{1}\left(\mu x+(1-\mu) y \in[0,1]^{d}\right)|x-y| \\
& \exp \left(-\alpha|x-y|^{d}\right) \mathrm{d} \mu \mathrm{d} x \mathrm{~d} y .
\end{aligned}
$$

By the substitution $x=z+y$ we get

$$
\begin{aligned}
e_{\lambda, b} & =\frac{\lambda^{2}}{2} \int_{\mathbb{R}^{d}} \int_{\mathbb{R}^{d}} \int_{0}^{1} \mathbb{1}\left(\mu z+y \in[0,1]^{d}\right)|z| \exp \left(-\alpha|z|^{d}\right) \mathrm{d} \mu \mathrm{d} z \mathrm{~d} y \\
& \stackrel{\text { Fubini }}{=} \frac{\lambda^{2}}{2} \int_{\mathbb{R}^{d}} \int_{0}^{1} \int_{\mathbb{R}^{d}} \mathbb{1}\left(y \in[0,1]^{d}-\mu z\right)|z| \exp \left(-\alpha|z|^{d}\right) \mathrm{d} y \mathrm{~d} \mu \mathrm{d} z \\
& =\frac{\lambda^{2}}{2} \int_{\mathbb{R}^{d}}|z| \exp \left(-\alpha|z|^{d}\right) \mathrm{d} z=d \kappa_{d} \lambda^{2} \int_{0}^{\infty} t^{d} \exp \left(-\alpha t^{d}\right) \mathrm{d} t,
\end{aligned}
$$

where last line of (A.5) is obtained by substitution of Euclidean coordinates by spherical coordinates. Here $\kappa_{d}=\pi^{d / 2} / \Gamma(d / 2+1)$ denotes the volume of the $d$-dimensional ball. Integration by parts leads to the claim

$$
\begin{aligned}
e_{\lambda, b} & =\frac{d \kappa_{d} \lambda^{2}}{2}\left(\left.\left(\frac{-t}{d \alpha} \exp \left(-\alpha t^{d}\right)\right)\right|_{0} ^{\infty}+\int_{0}^{\infty} \frac{1}{d \alpha} \exp \left(-\alpha t^{d}\right) \mathrm{d} t\right) \\
& =\frac{\kappa_{d} \lambda^{2}}{2 \alpha} \int_{0}^{\infty} \exp \left(-\alpha t^{d}\right) \mathrm{d} t=\frac{\kappa_{d} \lambda^{2}}{2 d \alpha^{1+\frac{1}{d}}} \Gamma\left(\frac{1}{d}\right) \\
& =\frac{2^{d-1+\frac{1}{d}} \lambda^{1-\frac{1}{d}} \pi^{\frac{1}{2 d}}}{b^{d+1} d\left(\int_{0}^{\arccos \left(1-\frac{1}{b}\right)} \sin ^{d}(t) \mathrm{d} t\right)^{1+\frac{1}{d}}} \frac{\left(\Gamma\left(\frac{d+1}{2}\right)\right)^{1+\frac{1}{d}} \Gamma\left(\frac{1}{d}\right)}{\Gamma\left(\frac{d}{2}+1\right)}
\end{aligned}
$$

\section{References}

[1] D. J. Aldous and J. Shun. Connected spatial networks over random points and a route-length statistic. Statistical Science, 25(3):275-288, 2010 . 
[2] A. Bertei, C. C. Chueh, J. G. Pharoah, and C. Nicolella. Modified collective rearrangement sphere-assembly algorithm for random packings of nonspherical particles: Towards engineering applications. Powder Technology, 253:311-324, 2014.

5 [3] Q. Cai, C. S. Adjiman, and N. P. Brandon. Modelling the 3D microstructure and performance of solid oxide fuel cell electrodes: Computational parameters. Electrochimica Acta, 56(16):5804-5814, 2011.

[4] S. N. Chiu, D. Stoyan, W. S. Kendall, and J. Mecke. Stochastic Geometry and its Applications. J. Wiley \& Sons, Chichester, 3rd edition, 2013.

[5] H. W. Choi, A. Berson, J. G. Pharoah, and S. B. Beale. Effective transport properties of the porous electrodes in solid oxide fuel cells. Proceedings of the Institution of Mechanical Engineers, Part A: Journal of Power and Energy, 225(2):183-197, 2011.

[6] M. B. Clennell. Tortuosity: a guide through the maze. Geological Society, London, Special Publications, 122(1):299-344, 1997.

[7] A. R. Conn, K. Scheinberg, and L. N. Vicente. Introduction to Derivative-free Optimization. SIAM, Society for Industrial and Applied Mathematics, Philadelphia, PA, 2009.

[8] P. Costamagna, P. Costa, and V. Antonucci. Micro-modelling of solid oxide fuel cell electrodes. Electrochimica Acta, 43(3):375-394, 1998.

[9] P. F. Felzenszwalb and D. P. Huttenlocher. Distance transforms of sampled functions. Theory of Computing, 8:415-428, 2009.

[10] G. Gaiselmann, M. Neumann, O. M. Pecho, T. Hocker, V. Schmidt, and L. Holzer. Quantitative relationships between microstructure and effective transport properties based on virtual materials testing. AIChE Journal, 60(6):1983-1999, 2014.

[11] GeoDict - open source software. www.geodict.com, 2014.

[12] J. Golbert, C. S. Adjiman, and N. P. Brandon. Microstructural modeling of solid oxide fuel cell anodes. Industrial $\&$ Engineering Chemistry Research, 47(20):7693-7699, 2008. 
[13] C. Hirsch, D. Neuhäuser, and V. Schmidt. Connectivity of random geometric graphs related to minimal spanning forests. Advances in Applied Probability, 45(1):20-36, 2013.

[14] L. Holzer, B. Iwanschitz, T. Hocker, L. Keller, O. M. Pecho, G. Sartoris, P. Gasser, and B. Muench. Redox cycling of Ni-YSZ anodes for solid oxide fuel cells: Influence of tortuosity, constriction and percolation factors on the effective transport properties. Journal of Power Sources, 242:179-194, 2013.

[15] L. Holzer, B. Münch, B. Iwanschitz, M. Cantoni, T. Hocker, and T. Graule. Quantitative relationships between composition, particle size, triple phase boundary length and surface area in nickel-cermet anodes for solid oxide fuel cells. Journal of Power Sources, 196(17):7076-7089, 2011.

[16] B. Kenney, M. Valdmanis, C. Baker, J. G. Pharoah, and K. Karan. Computation of tpb length, surface area and pore size from numerical reconstruction of composite solid oxide fuel cell electrodes. Journal of Power Sources, 189(2):1051-1059, 2009.

[17] J. F. C. Kingman. Poisson Processes, volume 3. Oxford University Press, Oxford, 1992.

[18] D. R. Kirkpatrick and J. D. Radke. A framework for computational geometry. In G. T. Toussaint, editor, Computational Geometry, pages 217-248. North-Holland, Amsterdam, 1985.

[19] S. Li. Concise formulas for the area and volume of a hyperspherical cap. Asian Journal of Mathematics and Statistics, 4(1):66-70, 2011.

[20] C. Metcalfe, O. Kesler, T. Rivard, F. Gitzhofer, and N. Abatzoglou. Connected three-phase boundary length evaluation in modeled sintered composite solid oxide fuel cell electrodes. Journal of the Electrochemical Society, 157(9):B1326-B1335, 2010.

[21] J. MÃÿller and R. P. Waagepetersen. Statistical Inference and Simulation for Spatial Point Processes. Chapman \& Hall/CRC, Boca Raton, 2004 . 
[22] B. Münch and L. Holzer. Contradicting geometrical concepts in pore size analysis attained with electron microscopy and mercury intrusion. Journal of the American Ceramic Society, 91(12):4059-4067, 2008.

[23] J. A. Nelder and R. Mead. A simplex method for function minimization. The Computer Journal, 7:308-313, 1965.

[24] J. Ohser and K. Schladitz. 3D Images of Materials Structures: Processing and Analysis. J. Wiley \& Sons, Weinheim, 2009.

[25] O. M. Pecho, O. Stenzel, P. Gasser, M. Neumann, V. Schmidt, T. Hocker, R. J. Flatt, and L. Holzer. 3D microstructure effects in Ni-YSZ anodes: Prediction of effective transport properties and optimization of redox-stability. Materials, 8(9):5554-5585, 2015.

[26] J. G. Pharoah, L. Handl, and V. Schmidt. The effect of electrode morphology on solid oxide fuel cell performance. ECS Transactions, 68:20372045, 2015.

[27] B. S. Prakash, S. S. Kumar, and S. T. Aruna. Properties and development of Ni/YSZ as an anode material in solid oxide fuel cell: A review. Renewable and Sustainable Energy Reviews, 36:149-179, 2014.

[28] N. Shikazono, D. Kanno, K. Matsuzaki, H. Teshima, S. Sumino, and N. Kasagi. Numerical assessment of SOFC anode polarization based on three-dimensional model microstructure reconstructed from FIB-SEM images. Journal of the Electrochemical Society, 157(5):B665-B672, 2010.

[29] O. Stenzel, O. M. Pecho, M. Neumann, V. Schmidt, and L. Holzer. Predicting effective conductivities based on geometric microstructure characteristics. AIChE Journal, in print, 2016.

[30] K. Thulasiraman and M. N. S. Swamy. Graphs: Theory and Algorithms. J. Wiley \& Sons, New York, 1992.

[31] J. Villanova, J. Laurencin, P. Cloetens, P. Bleuet, G. Delette, H. Suhonen, and F. Usseglio-Viretta. 3D phase mapping of solid oxide fuel cell YSZ/Ni cermet at the nanoscale by holographic X-ray nanotomography. Journal of Power Sources, 243:841-849, 2013. 
[32] J. R. Wilson, W. Kobsiriphat, R. Mendoza, H.-Y. Chen, J. M. Hiller, D. J. Miller, K. Thornton, P. W. Voorhees, S. B. Adler, and S. A. Barnett. Three-dimensional reconstruction of a solid-oxide fuel-cell anode. Nature Materials, 5(7):541-544, 2006. 